\title{
Reproducibility of new classification schemes for the pathology of ductal carcinoma in situ of the breast
}

\author{
Peter Bethwaite, Nicole Smith, Brett Delahunt, Diane Kenwright
}

\begin{abstract}
Aim-To compare the interobserver variation in the pathological classification of ductal carcinoma in situ of the breast using two recently proposed classification schemes.

Methods-11 pathologists classified a set of 25 cases of ductal carcinoma in situ chosen to reflect a range of lesions, using the traditional architectural classification together with the modified cytonuclear grading scheme of Holland $e t$ al and the Van Nuys classification scheme. Participating pathologists received a standard tutorial, written information, and illustrative photomicrographs before their assessment of the cases.

Results-Interobserver agreement was poorest when using the architectural scheme $(\kappa=0.44)$, largely owing to variations in classifying lesions with a mixed component of patterns $(\kappa=0.13)$. Agreement was better using the modified cytonuclear grading scheme $(\kappa=0.57)$, with most consistency achieved using the Van Nuys scheme $(\kappa=0.66)$. Most discordant results using the later scheme were due to inconsistency in assessing the presence or absence of luminal necrosis Conclusions-Both the new classification schemes assessed in this study were an improvement over the traditional architectural classification system for ductal carcinoma in situ, and resulted in more reproducible pathological assignment of cases. The Van Nuys classification scheme is easy to apply, even to small areas of carcinoma, resulting in acceptable interobserver agreement between reporting pathologists. Additional work will be required to arrive at a consensus definition of necrosis for cases in the non-highgrade group.
\end{abstract}

(f Clin Pathol 1998;51:450-454)

Keywords: breast; carcinoma in situ; classification

Ductal carcinoma in situ of the breast is a heterogeneous lesion, showing variability in biological behaviour, ${ }^{1}$ distribution within the breast, ${ }^{2}$ mammographic appearances, ${ }^{3}$ expression of oncogenes and hormone markers, ${ }^{45}$ and histological appearances. ${ }^{6}$ An uncommon cause of palpable lesions within the breast, ductal carcinoma in situ is now often identified in women participating in breast screening programmes owing to its frequent association with tissue microcalcification. Although forming around $20 \%$ of screen detected "cancers," the natural history of ductal carcinoma in situ is poorly understood, ${ }^{7}$ and the ideal treatment for women is controversial, ranging from limited local removal to mastectomy, resulting in a degree of over- or undertreatment. ${ }^{8}$

Along with the recognition of the heterogeneity of ductal carcinoma in situ has come a desire to identify those features which usefully predict clinical behaviour and therefore guide appropriate treatment. In addition to factors such as tumour size, resection margin involvement, $x$ ray appearances, and the expression of a number of biological markers, the histological appearances appear to correlate with clinical behaviour. ${ }^{910}$ Traditionally pathologists have classified ductal carcinoma in situ using morphological features, in particular the architectural pattern of the lesion. The major patterns recognised are: solid, comedo, cribriform, micropapillary, papillary, and clinging, with several less common patterns described (apocrine, neuroendocrine-like, secretory, and clear cell). ${ }^{6}$ Increasingly this traditional histological classification scheme has been found unsatisfactory, showing limited correlation with other prognostic markers, an inability to cope with mixed pattern lesions, and a marked degree of subjectivity in its application. ${ }^{11}{ }^{12}$

In an attempt to overcome the limitation of the traditional architectural classification scheme, various new systems have been proposed. ${ }^{11}$ These have shifted the focus from architecture to cytonuclear morphology, as first proposed by Lagios and colleagues over 15 years ago.$^{13}$ One classification scheme proposed by a European group of breast pathologists concentrates primarily on cytonuclear differentiation, with some secondary consideration given to one architectural feature (cell polarisation), to divide ductal carcinoma in situ into three groups: poorly differentiated, intermediate, and well differentiated. ${ }^{11}$ This European classification scheme has been found to correlate well with the mammographic appearances of ductal carcinoma in situ, ${ }^{3}$ the growth distribution of the carcinoma within the breast, ${ }^{2}$ and the expression of biological markers. ${ }^{4}$. In addition there appears to be a close association between the three grades of ductal carcinoma in situ and the grade of any associated infiltrating ductal carcinoma. ${ }^{14}$ This scheme has been adopted in the guidelines for reporting in the United Kingdom NHS breast screening programme, modified to emphasise nuclear grading alone. ${ }^{15}$ 
Table 1 Criteria for histological classification of ductal carcinoma in situ of the breast-architectural scheme (after Rogers ${ }^{6}$ )

Comedo-Presence of necrotic cellular debris in ductal or lobular spaces, usually centrally placed with viable cells in solid or perforated masses adjacent to the duct wall. Cells usually large with atypical nuclear features and conspicuous cytoplasm. Central calcification may be present, as may periductal fibrosis or chronic inflammation.

Solid-Neoplastic cells filling most or all of a duct-like space. Microlumina and papillary structures absent but calcifications may be present. Necrosis is not conspicuous although small randomly placed foci may be seen.

Cribriform - Fenestrated epithelial proliferation in which microlumina are formed in neoplastic epithelium bridging most or all of the duct lumen. Microlumina usually round/oval with smooth edges surrounded by a homogeneous uniformly distributed cell population.

Micropapillary - Duct-like spaces lined by neoplastic cells forming slender papillary fronds or arcuate formations protruding into the lumen. The papillae lack a fibrovascular core and are usually bulbous in shape, the tip broader than the base.

Papillary-A proportion of neoplastic cells arranged in papillary structures protruding into duct-like lumina supported by fibrovascular stromal cores. In part the papillae may fuse to form solid or fenestrated areas, but where a papillary component is present this is not classified as "mixed."

Clinging - A single or a few layers of neoplastic cells line the lumen of a duct-like space that is otherwise patent. A focal micropapillary pattern may be present, when this is $>10 \%$ the classification is "micropapillary."

Mixed - A combination of two or more patterns in separate duct-like spaces.

Other proposed classification schemes have included an assessment of the presence of necrosis following the observed association of luminal necrosis with increased rates of disease recurrence after local treatment. ${ }^{16}$ The "Van Nuys" system is one such scheme, ${ }^{17}$ a modification of the Nottingham classification of ductal carcinoma in $\operatorname{situ}^{18}$ whereby a high grade group is defined solely on the presence of high grade nuclear features, with the cases not classified as high grade being divided according to the presence or absence of necrosis. Retrospective application of this classification scheme to 425 women with ductal carcinoma in situ showed significant differences in local recurrence rates and disease-free survival between the three groups. $^{17}$

As well as providing useful clinical information on behaviour, the new proposed classification schemes for ductal carcinoma in situ must be easy to apply in routine pathology practice and be readily reproducible. In this paper we examine the consistency of reporting when applying the modified European group and the Van Nuys scheme to a series of ductal carcinoma in situ lesions.

\section{Methods}

Archival pathology material from Medical Laboratory (Wellington) and the Department of Anatomic Pathology (Wellington Hospital) were searched over a two year period and all cases coded to contain ductal carcinoma in situ of the breast, with or without an associated invasive component, were retrieved. From the 160 cases identified, 25 were selected by one of the investigators (PBB) as representing "typical" examples where the material had been well processed. Cases were not chosen on the basis of their ease or otherwise of classification but rather to reflect the range of common architectural patterns of ductal carcinoma in situ (comedo, solid, cribriform, micropapillary, clinging, and papillary) and to give representation of each of the subtypes in the new classification schemes. A new haematoxylin and eosin stained slide from each case was prepared to reflect uniform cutting and staining of material, and the slide covered with silver masking tape to reveal only the area of carcinoma for review. The area for review averaged $9 \mathrm{~mm}^{2}$, with any adjacent invasive component not visible to the assessor.

Eleven New Zealand pathologists who were experienced in, and regularly involved with, reporting breast pathology were recruited into the study. All were currently reporting ductal carcinoma in situ using the traditional architectural classification. All participating pathologists received a 45 minute tutorial from one of the investigators (PBB) on the diagnostic criteria for the classification schemes, supported by written information and a set of illustrative photomicrographs. The slide set was circulated to each participant, who anonymously evaluated each case using the current architectural method, as defined in standard publications ${ }^{6}$ (table 1). Participants were then asked to review the cases using the modified cytonuclear grading scheme of Holland et al, and re-review using the Van Nuys classification scheme. A summary of the diagnostic criteria for each of the new schemes is given in tables 2 and 3 .

Table 2 Criteria for histological classification of ductal carcinoma in situ of the breast- modified cytonuclear grading scheme of Holland et al ${ }^{1}$

\begin{tabular}{|c|c|c|c|}
\hline Criteria & $\begin{array}{l}\text { High grade } \\
\text { "Poorly differentiated" }\end{array}$ & $\begin{array}{l}\text { Low grade } \\
\text { "Well differentiated" }\end{array}$ & $\begin{array}{l}\text { Intermediate grade } \\
\text { "Intermediately differentiated" }\end{array}$ \\
\hline Nuclei & $\begin{array}{l}\text { - Pleomorphic } \\
\text { - Irregularly spaced } \\
\text { - Usually large } \\
\text { - Vary in size and shape } \\
\text { - Irregular contours } \\
\text { - Coarse clumped chromatin } \\
\text { - Prominent, sometimes } \\
\text { multiple nucleoli }\end{array}$ & $\begin{array}{l}\text { - Roughly spherical } \\
\text { - Centrally placed } \\
\text { - Usually small } \\
\text { - Smooth nuclear membrane } \\
\text { - Uniform fine chromatin } \\
\text { - Inconspicuous nucleoli }\end{array}$ & $\begin{array}{l}\text { - Mild pleomorphism } \\
\text { - Some variation in size and shape } \\
\text { - Irregularity of outline-lack the } \\
\text { monotony of low grade } \\
\text { - } 1-2 \text { nucleoli may be seen-not as } \\
\text { prominent as high grade } \\
\text { - N:C ratio often high }\end{array}$ \\
\hline Mitoses & $\begin{array}{l}\text { - Frequently present } \\
\text { - May be abnormal forms }\end{array}$ & - Uncommon & - Occasionally present \\
\hline Apoptosis & $\begin{array}{l}\text { - Apoptosis / individual cell } \\
\text { necrosis usually present }\end{array}$ & - Uncommon & - Uncommon \\
\hline Other features & $\begin{array}{l}\text { - Periductal fibrosis }+/- \\
\text { inflammatory cell infiltrate may } \\
\text { be present }\end{array}$ & - & - \\
\hline
\end{tabular}


Table 3 Criteria for histological classification of ductal carcinoma in situ-Van Nuys scheme (after Silverstein et al $^{17}$ )

\begin{tabular}{llll}
\hline Criteria & High grade & $\begin{array}{l}\text { Non-high grade with } \\
\text { necrosis }\end{array}$ & $\begin{array}{l}\text { Non-high grade without } \\
\text { necrosis }\end{array}$ \\
\hline $\begin{array}{l}\text { Cytonuclear } \\
\text { features }\end{array}$ & $\begin{array}{l}\text { Features of high grade } \\
\text { as in table } 2\end{array}$ & $\begin{array}{l}\text { Features of either low or } \\
\text { intermediate grade as in } \\
\text { table } 2\end{array}$ & $\begin{array}{l}\text { Features of either low or } \\
\text { intermediate grade as in } \\
\text { table } 2\end{array}$ \\
Intraduct necrosis & Present or absent & $\begin{array}{l}\text { Zone of necrosis } \\
\text { occupying }>10 \% \text { of the } \\
\text { diameter of the involved } \\
\text { duct }\end{array}$ & $\begin{array}{l}\text { Absence of necrosis or } \\
\text { involving }=10 \% \text { of the } \\
\text { diameter of the involved } \\
\text { duct }\end{array}$ \\
& &
\end{tabular}

Table 4 Distribution of agreement according to the internal majority opinion (\%) for each classification scheme: (A) architectural, (B) modified European group scheme, (C) Van Nuys scheme

\begin{tabular}{lccccc}
\hline $\begin{array}{l}\text { (A) Total response (\%) } \\
\text { Internal majority (n) }\end{array}$ & Comedo & Micropapillary & Solid & Cribriform & Mixed \\
\hline Comedo (6) & $\mathbf{7 5 . 8}$ & 4.5 & 3.0 & 3.0 & 13.6 \\
Micropapillary (2) & 0 & $\mathbf{6 3 . 7}$ & 0 & 13.6 & 22.7 \\
Solid (5) & 10.9 & 0 & $\mathbf{6 7 . 3}$ & 3.6 & 18.2 \\
Cribriform (10) & 7.3 & 1.8 & 3.6 & $\mathbf{6 6 . 4}$ & 20.9 \\
Mixed (2) & 0 & 9.1 & 9.1 & 18.2 & $\mathbf{6 3 . 6}$ \\
\hline
\end{tabular}

(B) Nuclear grade (\%)

\begin{tabular}{lclc}
\hline Internal majority $(n)$ & High & Intermediate & Low \\
\hline High (9) & $\mathbf{8 5 . 9}$ & 14.1 & 0 \\
Intermediate (11) & 11.6 & $\mathbf{8 1}$ & 7.4 \\
Low (5) & 0 & 21.8 & $\mathbf{7 8 . 2}$ \\
\hline
\end{tabular}

(C) Group (\%)

\begin{tabular}{lrcl}
\hline Internal majority (n) & $\begin{array}{l}\text { High } \\
\text { grade }\end{array}$ & $\begin{array}{l}\text { Non-high with } \\
\text { necrosis }\end{array}$ & $\begin{array}{l}\text { Non-high w/out } \\
\text { necrosis }\end{array}$ \\
\hline High grade (9) & $\mathbf{8 5 . 9}$ & 6.1 & 8.1 \\
Non-high with necrosis (5) & 12.7 & 70.9 & 16.5 \\
Non-high w/out necrosis (11) & 5.8 & 2.5 & $\mathbf{9 1 . 7}$ \\
\hline
\end{tabular}

Interobserver variation between the 11 pathologists was assessed by calculation of the kappa ( $\kappa)$ statistic, modified for multiple raters using the method Fleiss. ${ }^{19}$ The $\kappa$ statistic is independent of assumptions about the "true diagnosis" and is equal to zero if agreement between the raters is no better than chance, while $\kappa=1$ reflects perfect agreement.

\section{Results}

The distribution of agreement according to the internal majority opinion for the cases using each of the three classification schemes is given in table 4 .

\section{ARCHITECTURAL GRADING}

The majority diagnoses for the 25 cases reviewed by 11 pathologists were: comedo ( $\mathrm{n}=$ $6)$, micropapillary $(n=2)$, solid $(n=5)$, cribriform $(n=10)$, and mixed $(n=2)$. The overall $\kappa$ statistic for agreement in categorisation between the pathologists by architectural pattern was 0.44 . Interobserver agreement was greatest for the category "comedo" $(\kappa=0.60)$ and greatest variation was seen in categorising cases as "mixed" $(\kappa=0.13)$.

\section{MODIFIED EUROPEAN PATHOLOGISTS/NHSBSP} SCHEME

The majority diagnoses on the cases using the modified Holland cytonuclear grading scheme were: poorly differentiated (high grade) $(\mathrm{n}=$
9), intermediate differentiation (intermediate grade) $(n=10)$, and well differentiated (low grade) $(n=6)$. The overall interobserver variation was less marked compared with the traditional classification $(\kappa=0.57)$. Agreement was best when assigning cases to high nuclear grade $(\kappa=0.68)$ and poorest for the intermediate category $(\kappa=0.45)$.

\section{VAN NUYS CLASSIFICATION}

The majority diagnoses applying this scheme were: high grade $(n=9)$, non-high grade with necrosis $(n=5)$, and non-high grade without necrosis $(n=11)$. The overall interobserver variation was less than with the two previous classification schemes $(\kappa=0.66)$, with most variation seen in deciding whether non-high grade cases had evidence of luminal necrosis or not $(\kappa=0.50)$.

\section{Discussion}

The current method of classifying ductal carcinoma in situ, still used by many pathologists in routine surgical pathology practice, relies on an assessment of the overall architecture of the lesion including the presence or absence of comedo necrosis. In a large national external quality assurance scheme in the United Kingdom, consistency of reporting ductal carcinoma in situ by up to 250 participating pathologists was found to be low. ${ }^{12}$ The overall $\kappa$ statistic (comparing all responses to an internal majority opinion) for ductal carcinoma in situ in this study was only 0.23 . In the current smaller study, the overall $\kappa$ was higher (0.44), although this was calculated on the basis of overall responses rather than assuming one correct response derived from the internal majority opinion. In the United Kingdom study, as in the current study, agreement was best for comedo ductal carcinoma $(\kappa=0.44)$ and worst for the mixed patterns (0.06). Douglas-Jones and colleagues have recently reported on an assessment of 180 cases of screen detected infiltrating ductal carcinoma of the breast with independent classification of the associated ductal carcinoma in situ by two experienced observers using several different schemes. ${ }^{20}$ In this study disagreement between the two observers was most marked when assessing architectural patterns of ductal carcinoma in situ (34.4\% of cases). All three studies highlight one of the significant difficulties with the traditional classification scheme-the frequent occurrence of mixed patterned lesions. Lacking clear guidelines, some pathologists will use the "mixed" category for all true mixed lesions, others will feel that even a small component of comedo carcinoma in a mixed lesion overrides that diagnosis, while others will report the most prevalent architectural pattern. In the present study, where participants had only a small proportion of the total lesion to review, a mixture of morphological patterns within a few adjacent "ducts" was treated differently by the pathologists. Even when arbitrary rules are defined, classification of mixed cases becomes dependent on an assessment of the percentages of the different 


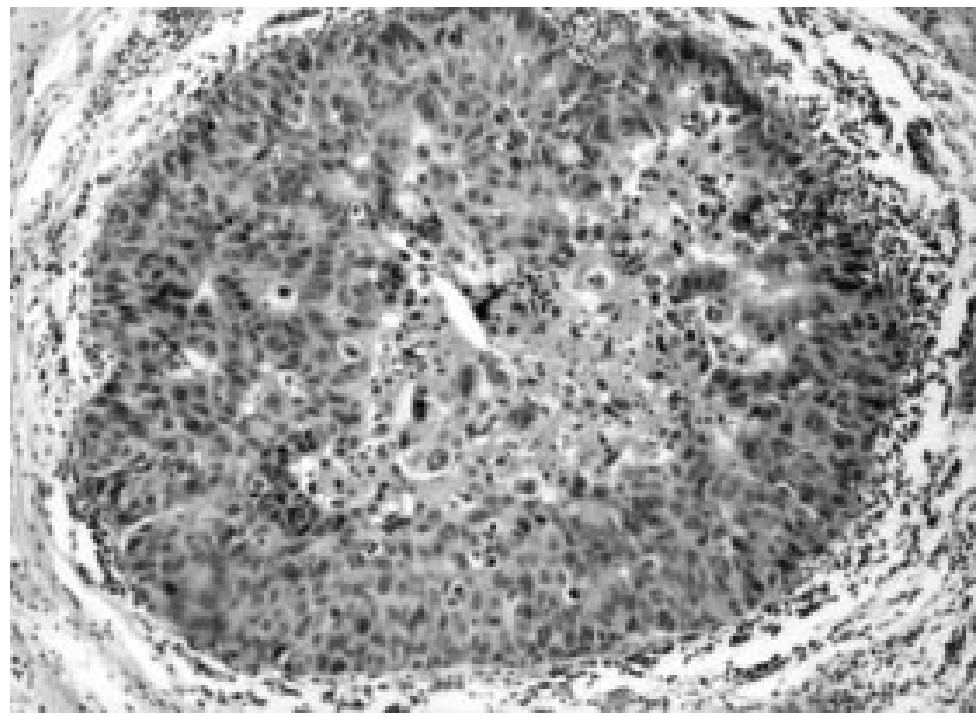

Figure 1 Non-high-grade ductal carcinoma with necrosis (Van Nuys scheme). All participating pathologists agreed on the presence of necrosis in this case, with a prominent area of central debris containing many pyknotic nuclei. Haematoxylin and eosin, $\times 134$.

components, the scheme soon becoming complex and unwieldy. ${ }^{20}$

Dissatisfaction with the traditional pathological classification of ductal carcinoma in situ has led to attempts at producing improved schemes that are both reproducible and clinically useful. Proposals have included the use of a dichotomous system of "comedo" and "non-comedo" groups, ${ }^{6}$ as pathologists appear to recognise this distinction reproducibly. ${ }^{12}$ This is rather an oversimplification since comedo-type necrosis can be seen in a proportion of "non-aggressive" lesions whether defined by nuclear grade, biological marker expression, or local recurrence risk. ${ }^{18} \mathrm{~A}$ classification of ductal carcinoma in situ into large cell and small cell types suffers from similar limitations. Other variations on these

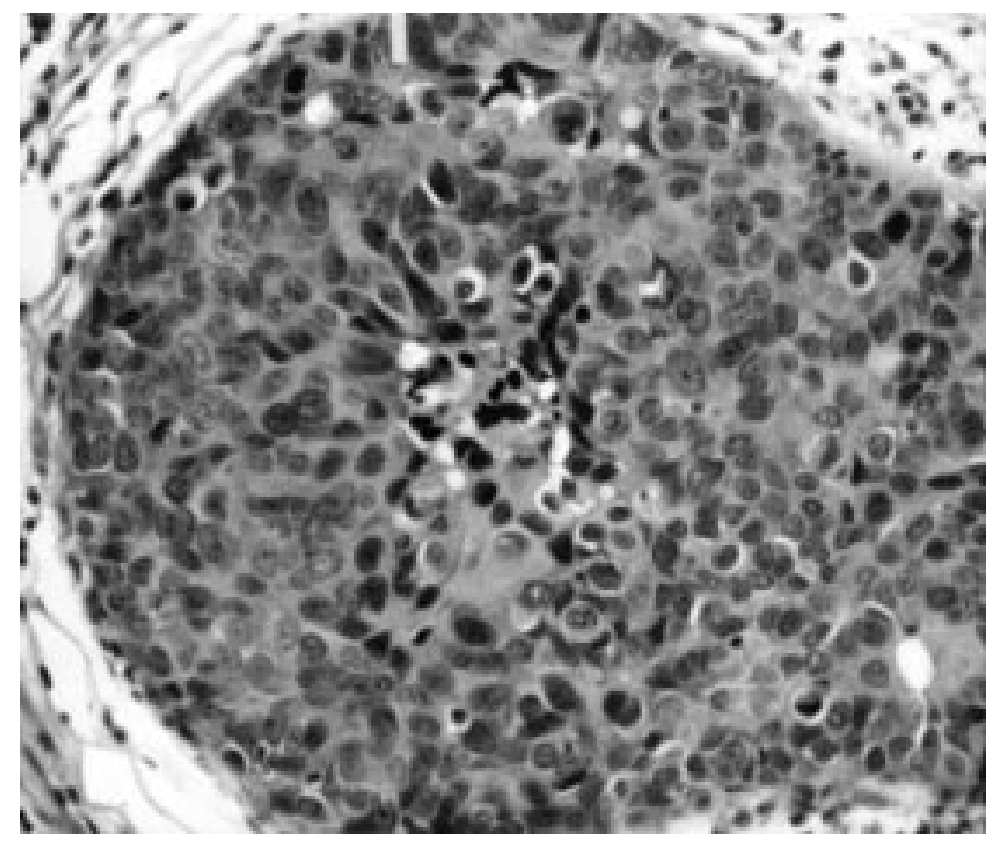

Figure 2 Non-high-grade ductal carcinoma. This duct shows early central necrosis, occupying less than $10 \%$ of the duct area but with greater than five pyknotic nuclei. This would be called positive for necrosis by some observers. Haematoxylin and eosin, $\times 268$. schemes have been proposed and these are fully reviewed in a recent publication. ${ }^{11}$

The importance of nuclear grade in ductal carcinoma in situ lesions was outlined by Lagios and colleagues, who ventured a classification based on architecture, necrosis, and cytonuclear features that appeared to relate to the behaviour of the lesion. ${ }^{13}{ }^{16}$ The European Group of Pathologists' classification represented an attempt at a more formal definition of the subtyping criteria for ductal carcinoma in situ, making use of cytonuclear grade after reviewing a large number of cases. ${ }^{11}$ This scheme includes a secondary feature describing cellular polarisation around lumina and over papillary surfaces; these changes can be somewhat subtle and open to variable interpretation. There is evidence from one study that inclusion of polarity assessment results in reclassification of less than $2 \%$ of cases, ${ }^{20}$ and these criteria have not been included in the ductal carcinoma classification recommended for pathology reporting in the NHS breast screening programme, which otherwise uses the European group nuclear grading scheme. ${ }^{15}$

Our study shows that when using the modified European group method agreement between pathologists is better than with the architectural method. Not surprisingly the participating pathologists were most consistent in assigning cases to the poorly differentiated (high cytonuclear grade) category. The intermediate group was the most popular category, but consistency in assigning cases to this group was poor. A problem with any three grade categorisation is the tendency for "central creep," whereby the line of least resistance is to use the intermediate or moderate category. This is to some degree implicit in the algorithm for using the modified European group method, which first tries to fit cases into the high or low group, with the middle grade acting as a default response. In the study reported by DouglasJones and coworkers, discordant cytonuclear grades were assigned by the two observers in 53 of the 180 cases, almost two thirds of the disagreements involving the distinction between low and intermediate cytological grade. ${ }^{20}$

The Van Nuys classification scheme recognises that both high versus low nuclear grade ductal carcinoma in situ, and the presence versus the absence of comedo-type necrosis are features associated with prognosis. ${ }^{17}$ Of the three schemes assessed, the Van Nuys showed the greatest reproducibility between pathologists. Rather than judge a spectrum of grades, in the Van Nuys scheme the pathologist is asked to make one or two dichotomous choices-high nuclear grade versus other nuclear grade, and for the second, the presence or absence of necrosis. The pathologists were reasonably consistent in recognising high cytonuclear grade features, but were less consistent in deciding on the presence or absence of necrosis. In the description of the Van Nuys scheme, "necrosis" was defined as comedo in type, with no requirement for a minimum amount to be present, although "occasional desquamated or individually necrotic cells 
(are) ignored."17 In a proportion of cases of ductal carcinoma, a minor component of central necrosis is seen, with or without foam cell change. In our study an arbitrary definition of necrosis was employed whereby the zone of necrotic debris should occupy more than 10\% of the diameter of the involved duct (fig 1). Our findings support those of Douglas-Jones et al who reported lower levels of disagreement between two observers using the Van Nuys scheme $(21.1 \%)$ than with cytonuclear grade $(29.4 \%)$ or architectural classifications $(34.3 \%) .{ }^{20}$ These investigators defined necrosis as "eosinophilic debris containing five or more pyknotic nuclei" with no requirement for a minimum number of duct spaces to be involved (fig 2). In the study by Douglas-Jones et al, disagreement was equally split between assigning cases to high versus non-high grade cytology and on the presence or absence of necrosis.

The protocol used in studies of this type does not reflect the everyday clinical situation faced by reporting pathologists. The observers were focused on one small area of the material, with only well presented and stained preselected material considered in the rounds. Under these ideal conditions the reproducibility figures are likely to be overrepresentations of what can be achieved under the usual reporting situations. This may explain the poorer reproducibility for architectural grading in the United Kingdom study, where classification was made on the whole slide area, with many more pathologists contributing. ${ }^{12}$ Although the presence of mixed nuclear grading is less of a problem than mixed architectural patterns within the same lesion, not all cases are pure as to cytonuclear grade ${ }^{11}$; these problems are unlikely to be apparent when small selected areas of a lesion are examined. The number of cases examined in the present study is considerably less than the 180 screen detected lesions studied by DouglasJones et al, where a greater diversity of lesions could be considered. However, our study compared 11 observers rather than the dual observers of the Douglas-Jones investigation, thereby improving the precision and validity of the $\kappa$ values obtained.

The results of our study suggest that improvements can be made in the reproducibility of the pathological classification of ductal carcinoma in situ lesions. Both of the new schemes assessed in this study represent advances over the traditional classification model by defining groups of lesions that correlate well with other prognostic factors, and for the Van Nuys scheme apparently with clinical behaviour. The value of the European pathologists' scheme in predicting risk of local recurrence must await trials of treatment of these lesions by local excision. Our findings support the conclusions of Douglas-Jones et al that the Van Nuys classification scheme is easy to apply, even to small areas of ductal carcinoma, resulting in acceptable interobserver agreement between reporting pathologists. Additional work will be required to arrive at a consensus definition of necrosis for non-high grade cases, although a definition based on the presence of necrotic debris containing five or more pyknotic nuclei appears easier to apply than one based on a minimum proportion of the duct area involved by eosinophilic necrosis.

1 Holland R, Peterse J, Mills R, et al. Ductal carcinoma in situ: proposal for a new classification. Semin Diagn Pathol 1994;11:167-80.

2 Faverly D, Burgers L, Bult P, et al. Three-dimensional imagng of mammary ductal carcinoma in situ: clinical implications. Semin Diagn Pathol 1994;11:193-8.

3 Holland R, Hendriks J. Microcalcifications associated with ductal carcinoma in situ: mammographic-pathologic correlation. Semin Diagn Pathol 1994;11:181-92.

4 Bobrow L, Happerfield L, Gregory W, et al. The Bobrow L, Happerfield L, Gregory W, et al. The
classification of ductal carcinoma in situ and its association with biological markers. Semin Diagn Pathol 1994;11:199207.

5 Leal C, Schmitt F, Bento M, et al. Ductal carcinoma in situ of the breast: histologic categorization and its relationship to ploidy and immunological expressions of hormone receptors, p53 and c-erbB-2 protein. Cancer 1995;75: 2123-31.

6 Rogers L. Carcinoma in-situ. In: Page D, Anderson T, eds. Diagnostic histopathology of the breast. Edinburgh: Churchill Livingstone, 1987:157-92.

7 Morrow M. The natural history of ductal carcinoma in situ. Cancer 1995;76:1113-14.

8 van Dongen J, Holland R, Peterse J. Ductal carcinoma in-situ of the breast: second EORTC consensus meeting. Eur $\mathcal{F}$ Cancer 1992;28:626-9.

9 Bellamy C, McDonald C, Salter D, et al. Noninvasive ductal carcinoma of the breast: the relevance of histologic categorization. Hum Pathol 1993;24:16-23.

10 Lagios M. Heterogeneity of duct carcinoma in situ (DCIS): relationship of grade and subtype analysis to local recurrence and risk of invasive transformation. Cancer Lett 1994;90:97-102.

11 Mills R. Classification of ductal carcinoma in situ of the breast. Adv Anat Pathol 1996;3:114-29.

12 Sloane J, Ellman R, Anderson T, et al. Consistency of histopathological reporting of breast lesions detected by screening: findings of the UK national external quality assessment (EQA) scheme. Eur f Cancer 1994;30:1414-19.

13 Lagios M, Westdahl P, Margolin F, et al. Ductal carcinoma in situ. Relationship of extent of noninvasive disease to the frequency of occult invasion, multicentricity, lymph node metastases and short-term treatment failures. Cancer 1982; 50:130

14 Lampejo O, Barnes D, Smith P, et al. Evaluation of infiltrating ductal carcinomas with a ductal carcinoma component: correlation of the histologic type of the in situ component with grade of the infiltrating component. Semin Diagn Pathol 1994;11:215-22.

15 National Coordinating Group for Breast Screening Pathology. Pathology reporting in breast cancer screening, 2nd ed. Sheffield: NHS Breast Screening Programme, publication No 3, 1995.

16 Lagios M, Margolin F, Westdahl P, et al. Mammographically detected duct carcinoma in situ: frequency of local recurrence following tylectomy and prognostic effect of recurrence following tylectomy and prognostic effect of

17 Silverstein M, Poller D, Waisman J, et al. Prognostic classification of breast ductal carcinoma-in-situ. Lancet 1995;345:1154-7.

18 Poller D, Silverstein M, Galea M, et al. Ductal carcinoma in situ of the breast: a proposal for a new simplified histological classification association between cellular proliferation and c-erbB-2 protein expression. Mod Pathol 1994;7:257-

19 Fleiss J. The measurement of inter-rater agreement. In: Statistical methods for rates and proportions. New York: John Wiley and Sons, 1981:212-36.

20 Douglas-Jones A, Gupta S, Attanoos R, et al. A critical appraisal of six modern classifications of ductal carcinoma in situ of the breast (DCIS): correlation with grade of associated invasive carcinoma. Histopathology 1996;29:397409. 